Revue Française de Civilisation Britannique

\title{
La difficile conquête du droit à l'information
}

The Freedom of Information Act: an Uphill Battle

Jean-Claude Sergeant

\section{(2) OpenEdition}

Journals

Édition électronique

URL : http://journals.openedition.org/rfcb/704

DOI : $10.4000 /$ rfcb.704

ISSN : 2429-4373

Éditeur

CRECIB - Centre de recherche et d'études en civilisation britannique

Édition imprimée

Date de publication : 1 février 2002

Pagination : 65-77

ISBN : 2-911580-13-3

ISSN : 0248-9015

Référence électronique

Jean-Claude Sergeant, "La diffıcile conquête du droit à l'information», Revue Française de Civilisation Britannique [En ligne], XI-3 | 2002, mis en ligne le 21 mars 2016, consulté le 22 avril 2019. URL : http:// journals.openedition.org/rfcb/704; DOI : 10.4000/rfcb.704

Ce document a été généré automatiquement le 22 avril 2019

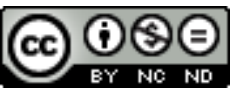

Revue française de civilisation britannique est mis à disposition selon les termes de la licence Creative Commons Attribution - Pas d'Utilisation Commerciale - Pas de Modification 4.0 International. 


\section{La difficile conquête du droit à l'information}

The Freedom of Information Act: an Uphill Battle

Jean-Claude Sergeant

1 Si le XIX siècle a été marqué par les luttes qui ont permis l'élimination progressive des obstacles à la diffusion de l'information - les célèbres «Taxes on Knowledge» dont la dernière fut abolie en 1861 - le dernier quart du $\mathrm{XX}^{\mathrm{e}}$ siècle a connu un combat non moins important qui avait pour enjeu le droit à l'information, c'est-à-dire le droit pour tout citoyen de consulter des documents officiels auparavant inaccessibles au public et les gisements documentaires détenus par les acteurs publics.

2 Complément indispensable du fonctionnement d'une démocratie adulte, la capacité du citoyen de s'informer par lui-même des raisons des choix politiques opérés par les dirigeants ou de vérifier les modes de fonctionnement des agences para-publiques qui ont proliféré depuis l'époque Thatcher, semblait être l'antidote de la désaffection de l'électorat qui a atteint des niveaux records lors des élections de juin 2001 (60\% de participation, taux le plus faible depuis 1918).

3 Le rôle central attribué par les médias aux conseillers en communication, les fameux spin doctors identifiés à Alistair Campbell, directeur du service de presse du premier ministre, n'avait pas non plus contribué à accréditer auprès des électeurs le concept de transparence. La gestion politique semblait trop souvent se ramener à un art de la communication privilégiant la présentation par rapport à la substance d'un choix argumenté.

4 Producteur et contrôleur de l'essentiel de l'information nationale, à l'exception de l'information économique qui trouve son origine dans les entreprises relevant toutes aujourd'hui du secteur privé, l'État ou, pour mieux le dire, les pouvoirs publics, se sont trouvés dans l'obligation de répondre à la volonté des citoyens d'être mieux informés des raisons ayant présidé au choix de telle politique susceptible de les affecter. Cette demande d'accès à l'information institutionnelle était d'ailleurs exprimée explicitement par le public. À l'occasion d'un sondage portant sur les valeurs morales des Britanniques 
réalisé par l'institut MORI, $85 \%$ des répondants plaçaient l'élargissement de l'accès à l'information gouvernementale en tête de leurs souhaits, ce qui en faisait leur revendication prioritaire parmi un ensemble de dix propositions présentées par l'institut de sondage ${ }^{1}$. On observe l'évolution des mentalités sur ce point en se reportant aux textes, peu nombreux, consacrés au sujet à la fin des années soixante-dix. Dans l'un des opuscules publiés par la Fabian Society intitulé Open Up!, l'auteur, naturellement partisan $\mathrm{du}$ desserrement du verrouillage de l'information officielle, pouvait encore écrire : « $L a$ question de l'accès à l'information officielle (Open Government) n'est naturellement pas de celles qui inspirent l'électorat", ajoutant, comme pour s'excuser, que "le fait que seule une minorité politisée et qui se fait entendre fasse campagne pour l'obtenir ne signifie pas que la question soit sans intérêt ${ }^{2} »$.

5 Sans doute plus attentif qu'hier au rôle des dirigeants dans la gestion des crises sanitaires (ESB, fièvre aphteuse) et à leur responsabilité en matière de sécurité (celle du transport ferroviaire, des centrales nucléaires par exemple), le public, mieux instruit des risques, n'accepte plus d'être placé devant le fait accompli. La légitimité des acteurs politiques est de plus en plus conditionnée par leur capacité à rendre compte de leurs décisions et de l'usage qu'ils ont fait de l'information qu'ils détenaient et dont, si l'on en croit Tony Benn, ils aimeraient conserver le monopole. Lors d'une conférence donnée en 1978, l'ancien ministre de Harold Wilson analysait en ces termes la réticence des responsables à partager l'information : «Permettre l'accès à l'information affaiblit la prérogative des ministres et des fonctionnaires qui ne sont jamais aussi puissants que lorsqu'ils sont seuls à connaître les décisions qui se préparent, les choix qui seront arrêtés et l'information qui s'y rattache. Comment dans ces conditions contester leurs avis ? ${ }^{3}$ ”

\section{Les années d'atermoiements}

6 Les pressions en faveur de l'élargissement de l'accès à l'information officielle ont commencé à se faire sentir dès les années soixante à l'occasion de l'examen du fonctionnement de l'administration centrale. Le rapport Fulton ${ }^{4}$ dénonçait, outre le mode de recrutement et le manque de professionnalisme de la haute fonction publique, son manque de transparence et son refus de permettre un plus large accès à ses dossiers. La création en 1967 du poste de Parliamentary Commissioner for Administration (PCA), souvent identifié à un Ombudsman à la mode britannique, ne pouvait constituer à elle seule, une réponse aux demandes des membres de la commission Fulton. Chargé de régler les litiges entre l'administré de base et les responsables de la machine administrative, le PCA, en dépit des pouvoirs d'investigation dont il disposait, était lié par le respect de la loi sur les secrets officiels (l'Official Secrets Act, OSA) dont la section 2 permettait à tout titulaire d'une autorité gouvernementale d'estampiller du sceau d'information protégée n'importe quel document officiel et, par là-même, de le soustraire à la demande du PCA.

7 C'est, en fait, la prise de conscience par les responsables politiques du statut excessif accordé à l'information officielle par la loi sur les secrets officiels, remontant à 1911, qui les a amenés à évoluer par rapport à la question de l'accès à ce type d'information. Le non-lieu prononcé en 1971 par le juge Caulfield dans l'affaire Scott/Aitken/Roberts relative à la divulgation de documents infirmant la position officielle du gouvernement lors de la guerre du Biafra, incita les responsables politiques de l'époque à instituer une commission dirigée par Lord Franks chargée de réfléchir à une réforme de la loi sur les secrets officiels. 
8 De fait, la demande de transparence concernant l'information officielle se confondait à l'époque, avec celle qui avait pour objectif la réforme du système de protection des informations sensibles. Les deux démarches visaient néanmoins des buts fondamentalement différents. S'agissant des secrets officiels, les réformateurs entendaient obtenir l'abrogation de la section 2 de la loi qui interdisait sous peine d'amende et/ou d'emprisonnement, la divulgation non autorisée de toute information à caractère officiel, sans pour autant que soit remise en cause la légitimité de la protection de catégories d'informations sensibles dûment répertoriées. Les partisans de la transparence situaient leur action à un autre niveau, leur objectif étant de permettre l'accès par le particulier à un ensemble d'informations dépourvues de caractère sensible et pourtant non divulguées.

9 Il n'en reste pas moins qu'en pratique, les réformateurs luttaient sur les deux fronts simultanément. Le rapport de la commission présidée par Lord Franks, centré sur la réforme de la section 2 de l'OSA ${ }^{5}$, leur donnait d'ailleurs en partie raison puisqu'il excluait du champ de la protection accordée aux informations officielles les notes et documents internes élaborés par les fonctionnaires à l'intention de leurs ministres. Toutefois, il n'appartenait pas à la commission de préconiser que ces éléments d'information fussent mis à la disposition du public.

10 Sans aller jusqu'à reprendre à son compte cette possibilité, le parti travailliste n'en inscrivait pas moins dans son programme électoral pour les élections d'octobre 1974 l'obligation pour les autorités publiques de justifier toute rétention d'information. Cet engagement se traduira par la circulaire de Douglas Allen, alors responsable de l'administration centrale laquelle, en juillet 1977, enjoignait à ses subordonnés dans les ministères d'opérer une distinction entre l'information factuelle dont ils disposaient et celle à caractère politique et de mettre la première dans le domaine public. Cette pressante incitation eut des effets limités puisqu'entre mai et octobre 1978, deux cent onze éléments d'information seulement avaient été rendus publics, essentiellement par le ministère de la Fonction publique et celui de l'Éducation et de la Science ${ }^{6}$.

11 L'initiative du directeur de l'administration centrale devenu par la suite Lord Croham, fut relayée au sein de la Chambre des Communes par l'action de certains parlementaires, appartenant pour la plupart au parti libéral, qui tentèrent de faire évoluer la situation par le biais de propositions de loi. Celle déposée par Clement Freud en 1979 parvint même à franchir le cap de la deuxième lecture, avant d'être emportée par la défaite du gouvernement Callaghan.

12 Les groupes de pression n'étaient d'ailleurs pas en reste. Justice, branche britannique de la Commission internationale des juristes, élabora en 1978 un code de bonne conduite ( Code of Practice ${ }^{8}$ ) qui, tenant compte des réticences des principaux détenteurs de l'autorité administrative et politique, se bornait à confier à l'Ombudsman le soin de réglementer un système posant comme principe la divulgation, éventuellement assortie d'un délai, de l'ensemble de l'information à la disposition des rouages gouvernementaux et administratifs. Ce principe était, bien entendu, assorti des clauses d'exemption classiques protégeant, notamment, des catégories d'information touchant la défense, les relations internationales, la sécurité intérieure et l'action de la justice.

13 Le projet échafaudé par Justice n'allait pas toutefois jusqu'à impliquer l'adoption d'une loi garantissant la liberté de l'accès à l'information officielle. Celui conçu par l'Outer Circle Policy Unit, centre de réflexion financé par le Rowntree Trust, n'hésitait pas, en revanche, à 
franchir le pas. Son projet, qui fit l'objet d'une proposition de loi ${ }^{9}$, fut d'ailleurs l'une des sources d'inspiration de Clement Freud dont l'initiative malheureuse a été évoquée plus haut.

14 Ce texte, qu'un spécialiste ${ }^{10}$ qualifie de "rationaliste " parce qu'il le juge fondé sur des catégories permettant de distinguer à coup sûr l'information authentique de celle contaminée par le préjugé et la manipulation, avait pour objectif de permettre le rééquilibrage entre la haute administration et le gouvernement détenteurs du monopole de l'information, et le Parlement, supposé contrôler le gouvernement, mais incapable d'exercer effectivement ce contrôle, n'ayant pas accès à une partie de l'information officielle confisquée par l'exécutif et l'administration à son service. Il est à noter que, comme dans le projet conçu par Justice, aucun recours judiciaire n'était prévu dans la proposition émanant de l'Outer Circle Policy Unit qui s'en remettait également à l'autorité du Parliamentary Commissioner pour administrer le système et arbitrer les conflits éventuels.

15 Le gouvernement travailliste ne pouvait rester insensible à ces pressions relayées par le comité directeur du parti (National Executive Committee) et soutenues par une partie importante de sa base parlementaire. D'autres priorités politiques mobilisaient, toutefois, l'équipe gouvernementale dirigée par James Callaghan qui finit pourtant par publier en 1979 un Livre vert ${ }^{11}$ qui, tout en marquant l'accord du gouvernement s'agissant du principe d'un code de bonne conduite, n'en exprimait pas moins une certaine tiédeur par rapport à une loi élargissant l'accès à l'information officielle, jugée trop coûteuse et susceptible de perturber l'équilibre des pouvoirs constitutionnels.

16 Si un système de partage de l'information était, dans l'esprit du gouvernement, de nature à renforcer la démocratie parlementaire, un tel système, précisait le préambule du document, ne pouvait qu'être mis en œuvre de façon progressive, tout en tenant compte du mode de fonctionnement des institutions constitutionnelles. Il devait, en outre, être suffisamment évolutif pour intégrer les acquis de l'expérience ${ }^{12}$. En d'autres termes, le gouvernement de l'époque s'avançait avec une prudence calculée dans une voie qu'il n'explorait, de toute évidence, qu'à contrecœur.

\section{Les premiers pas de la réforme}

17 Le nouveau gouvernement conservateur issu des élections de 1979 s'est très vite trouvé enfermé dans un jeu de contradictions qui excluait toute avancée majeure en matière de transparence de l'information officielle. Si Mme Thatcher a pu, un temps, passer pour la championne de l'ouverture des dossiers détenus par les services administratifs et ministériels, elle s'est très vite repliée sur des positions restrictives protégeant l'action de l'État, dictées par sa conception de l'autorité incontestable du gouvernement et la nécessité de lutter contre le terrorisme lié au problème nord-irlandais. Le refus de hauts fonctionnaires de témoigner devant la commission d'enquête nommée pour établir la responsabilité des différents protagonistes impliqués dans l'affaire Westland (1986) illustre parfaitement le principe défendu au sommet de l'État de l'immunité de ses serviteurs.

18 C'est essentiellement dans le domaine de la protection des secrets officiels que les gouvernements Thatcher ont laissé leur marque. Après un premier essai infructueux qui a amené le retrait en 1979 d'un projet de loi intitulé Protection of Official Information, le 
gouvernement parvint à faire aboutir en 1989 une réforme de la loi de 1911 relative aux secrets officiels.

19 En dépit des déclarations du ministre de l'Intérieur à l'époque lors de la présentation du projet de loi pour qui cette réforme constituait «une avancée importante, sans précédent depuis la Seconde guerre mondiale, dans la voie d'une plus grande transparence ${ }^{13}$, rien dans le nouveau texte ne permettait de faciliter l'accès à l'information officielle, sauf à considérer que la limitation de sa protection à six grandes catégories assez nettement définies allait dans le sens d'une transparence renforcée.

20 Pour sa part, Sir Robert Armstrong, en sa qualité de responsable de l'administration centrale, rappelait en 1985 aux hauts fonctionnaires leur devoir de loyauté à l'égard de leurs ministres. Les serviteurs de l'État qu'ils étaient devaient collaborer dans un climat de confiance avec les membres du gouvernement qui, collectivement, incarnaient la Couronne.

21 Si l'administration centrale restait globalement retranchée derrière les dispositifs pérennisant le secret officiel, la situation évoluait sur d'autres fronts. En 1984, le Parlement adoptait la loi protégeant les données personnelles (Data Protection Act) qui permettait aux particuliers de consulter et, éventuellement, de demander la rectification, des dossiers informatisés les concernant. Il s'agissait, en fait, pour le gouvernement de mettre sa législation en conformité avec la réglementation européenne mais ce premier texte n'en marquait pas moins la reconnaissance d'un droit de regard sur une information jusqu'alors détenue sans garantie de contrôle par des services administratifs ainsi que par des organisations publiques ou privées.

22 La loi régissant l'accès à l'information détenue par les collectivités territoriales (Local Government [Access to Information]) Act), adoptée en 1985, constitue une étape plus significative encore en matière de transparence. Qualifiée d'«instrument législatif remarquable " par l'un des meilleurs spécialistes de la question ${ }^{14}$, cette loi non seulement rendait publiques les séances des conseils ainsi que les réunions de leurs différentes commissions, mais autorisait la consultation des documents, études et rapports relatifs à ces séances. Le gouvernement avait accordé au niveau local ce que beaucoup réclamaient à l'échelon central.

23 Il appartiendra au gouvernement de John Major de desserrer le carcan du contrôle exclusif de l'information officielle. Ayant souhaité responsabiliser l'administration et les prestataires de services publics au moyen de "Chartes des citoyens" permettant à l'usager de demander des comptes aux autorités publiques et imposant à ces dernières l'affichage de leurs objectifs et de leurs performances, John Major ne pouvait longtemps refuser d'appliquer à l'administration centrale et aux départements ministériels ce qu'il avait érigé en principe pour les échelons inférieurs du système. Un engagement en faveur d'un plus large accès à l'information officielle figurait, du reste, dans le manifeste conservateur pour les élections de 1992.

24 En assignant au Chancelier du duché de Lancastre, William Waldegrave, la mission de promouvoir une plus grande transparence de l'information, John Major indiquait qu'il entendait respecter son engagement. La publication de la liste des comités permanents du Cabinet et de leur composition (mai 1992) fut saluée comme un geste encourageant. Mme Thatcher s'était bornée à révéler l'identité des quatre principaux comités sans toutefois en préciser la composition. Son prédécesseur s'était, pour sa part, toujours refusé à faire connaître la liste de ces comités généraux permanents au motif que l'information serait 
de nature à attiser la curiosité plus qu'elle ne pourrait la satisfaire. John Major n'était toutefois pas allé jusqu'à promettre d'informer la presse, sinon a posteriori, de la création des comités ad hoc souvent plus nombreux que les comités permanents.

La porte avait été entrouverte selon la formule de l'éditorial du Times (20 mai 1992). Ce premier gage de la volonté d'ouverture qui animait incontestablement John Major allait être amplifié par le Livre blanc sur la transparence gouvernementale (Open Government) publié en juillet 1993.

\section{Le Code d'accès à l'information}

26 Posant comme principe que la transparence de l'action gouvernementale était la condition première du fonctionnement efficace d'une démocratie et, qu'en conséquence, les citoyens devaient pouvoir avoir connaissance de l'information sur laquelle reposait la conduite de l'action des responsables, le Livre blanc proposait de n'opposer un refus aux demandes d'accès à l'information que dans les seuls cas pour lesquels la rétention de l'information pouvait être justifiée. Il importait donc de dresser la liste exhaustive des cas d'exemption, c'est-à-dire des situations où le principe de divulgation ne s'appliquait pas. De fait, l'inventaire de ces cas allait constituer l'essentiel du Code d'accès à l'information publié en 1994 et révisé en 1997.

27 La démarche du gouvernement pouvait, en outre, paraître singulière en ce sens que, contrairement à la pratique adoptée par les gouvernements étrangers qui avaient reconnu un droit à l'information, le Livre blanc n'a pas débouché sur une loi fixant les conditions d'exercice de ce droit mais sur un code qui définissait les modalités d'accès à l'information. En d'autres termes, aucun recours ne pouvait être introduit devant un tribunal par un citoyen auquel la communication d'un document aurait été refusée. Le gouvernement avait donc renoncé à consacrer un droit d'accès à l'information officielle, préférant s'en tenir à la mise en œuvre d'un dispositif organisant cet accès.

28 Le code ne se limitait d'ailleurs pas à la définition de l'accès par un particulier à un document spécifique. Il prévoyait, en outre, la mise dans le domaine public de données factuelles et d'éléments d'analyse ayant servi à l'élaboration de décisions gouvernementales et jugés importants par les responsables politiques dans la perspective de contextualisation de leurs choix. Enfin, le code prescrivait la diffusion automatique des règlements, manuels explicatifs et autres documents internes émanant des services administratifs et des agences déléguées.

29 Ces principes étant établis, le code dressait l'inventaire des catégories d'informations protégées, c'est-à-dire n'entrant pas dans le champ d'application de l'obligation de divulgation. Étaient nécessairement soustraites à cette obligation les informations touchant la défense, la sécurité nationale, ainsi que les informations confidentielles adressées par les gouvernements et tribunaux étrangers ou émanant d'organisations internationales. Etait en outre proscrite la divulgation d'informations susceptibles d'altérer (harm) la franchise (candour) des débats au sein du Cabinet et de ses comités. Il s'agissait, en l'occurrence, de protéger la confidentialité des échanges entre membres du Cabinet et de ses comités et d'interdire l'accès aux documents, spéculatifs ou non, ayant servi à l'élaboration des politiques gouvernementales.

30 L'ensemble des informations concernant les actions judiciaires dont la divulgation serait susceptible d'affecter le cours entrait, en outre, et fort légitimement, dans la catégorie 
des informations « exemptées ", ainsi que celles relatives à l'emploi et à la promotion des personnels dépendant de l'État ou des collectivités publiques. C'est, au total, une quinzaine de catégories d'informations protégées qui se trouvent recensées dans le code d'accès à l'information officielle, y compris celles concernant une décision gouvernementale imminente et dont la communication serait jugée prématurée.

31 Il va de soi que même si le recours devant un tribunal était exclu en cas de refus de communication de l'information requise, le système mis en place avait prévu des voies d'appel. En l'espèce, c'est le Parliamentary Commissioner for Administration qui était chargé d'arbitrer les litiges. Son champ d'intervention était, toutefois, limité. Les recours concernant l'information détenue par la Banque d'Angleterre, les services de renseignement (MI5 et MI6), le service d'écoute de Cheltenham (GCHQ) ou par le Cabinet n'avaient aucune chance d'aboutir dans la mesure où les informations détenues par ces services ou organismes étaient exclues de son champ de compétences. En outre, le requérant éconduit ne pouvait s'adresser directement à l'Ombudsman, sa demande de recours devant nécessairement transiter par un membre de la Chambre des Communes.

32 Saisi par un MP, et convaincu du bien-fondé de la demande d'information rejetée par l'adminsitration concernée, l'Ombudsman ne pouvait qu'émettre des recommandations sans pouvoir exercer la moindre autorité sur l'administration récalcitrante. Bien que de portée limitée, le système a pourtant fonctionné et reste en vigueur en attendant l'application sans cesse retardée de la nouvelle loi sur la liberté d'information adoptée par le Parlement en 2000. En 1999, 4863 demandes d'accès à un document officiel ont été enregistrées contre 2493 en $1994^{15}$. Pour sa part l'Ombudsman a été saisi de trente-six recours en 1999, dont quinze seulement ont fait l'objet d'une enquête. Le Code d'accès à l'information, malgré ses insuffisances, s'était progressivement imposé comme une voie efficace d'accès à des informations considérées naguère comme taboues.

\section{"Your Right to Know"}

33 A la veille des élections de 1992, Neil Kinnock avait déclaré que l'une des premières décisions du gouvernement travailliste qu'il espérait voir sortir des urnes concernerait la mise en chantier d'un projet de loi consacrant le droit à l'information. Cinq ans plus tard cette priorité était réaffirmée dans le manifeste travailliste. Tony Blair devait tenir parole. Moins de huit mois après les élections de mai 1997 était publié un Livre blanc Your Right to Know ${ }^{16}$ - qui jetait les bases de la législation la plus avancée dans le domaine. Il ne s'agissait de rien moins, selon le premier ministre, auteur de la préface, que d'en finir avec la culture du secret propre à la tradition nationale et d'instaurer un changement radical dans les rapports entre gouvernants et gouvernés.

Le projet de législation devait s'appliquer à l'ensemble des organismes publics, y compris les agences déléguées (les fameuses quangos), ainsi qu'aux entreprises privées opérant dans le secteurs public (fourniture d'eau, d'énergie, etc.). Le texte reposait sur le principe selon lequel la communication sur simple demande d'informations et de documents factuels ne pouvait être refusée sauf à démontrer que leur divulgation était de nature à porter gravement atteinte (substantial harm) à l'intérêt public. Toutefois, s'agissant des avis transmis par les hauts fonctionnaires à leurs ministres, le refus de communication pouvait être justifié par la seule invocation d'une présomption d'atteinte, sans autre précision de gravité, à l'intérêt public. Le Livre blanc ne prévoyait plus que sept catégories d'informations protégées contre quinze répertoriées dans le code d'accès de 
1994. Se trouvaient ainsi soustraites à l'obligation de communication les informations relatives à la défense, à la sécurité nationale et aux relations internationales, celles concernant l'application de la $\operatorname{loi}^{17}$, les informations touchant à la vie privée des individus, celles touchant la sécurité du public et de l'environnement, les informations confidentielles transmises à un organisme public et, enfin, celles susceptibles d'affecter l'intégrité $d u$ processus décisionnel au sein du gouvernement. Les services de renseignement étaient en outre exclus du champ d'application du projet de loi envisagé ainsi que les dossiers concernant les fonctionnaires employés par le gouvernement.

35 Il était également prévu que le demandeur ${ }^{18}$ éconduit puisse faire appel d'un refus de communication en s'adressant au commissaire à l'information habilité à introduire un recours devant les tribunaux. Nommé par le premier ministre, ce commissaire aurait tous pouvoirs pour se faire remettre tous documents, y compris ceux non couverts par le projet et notamment les papiers du Cabinet et de ses comités. Le commissaire pourrait également user de son autorité pour ordonner la communication refusée et poursuivre devant un tribunal l'autorité publique récalcitrante. Le Livre blanc précisait également les délais de réponse accordés aux organismes saisis d'une demande ainsi que la gamme des coûts à la charge du demandeur, le tarif de base étant fixé à dix livres. La question du coût global de fonctionnement du système sera d'ailleurs l'un des arguments majeurs des adversaires du projet.

36 Le Livre blanc, élaboré par David Clark, Chancelier du duché de Lancastre, c'est-à-dire ministre sans portefeuille, fut accueilli avec enthousiasme par les commentateurs. Maurice Frankel, directeur de l'association Campaign for Freedom of Information, qui militait depuis longtemps en faveur de l'élargissement de l'accès à l'information, ne dissimulait pas sa satisfaction face à la véritable révolution que le Livre blanc allait opérer : «Dans l'ensemble, je pense qu'il s'agit là de mesures extrêmement positives, bien supérieures à ce qu'on imaginait pouvoir être proposé par un gouvernement britannique. ${ }^{19}$

\section{Le recul}

37 L'enthousiasme n'était pourtant pas partagé par tous dans les rangs du gouvernement et de la haute administration. L'idée d'avoir à démontrer l'atteinte grave à l'intérêt public pour justifier un refus consternait les fonctionnaires de l'administration centrale habitués à la discrétion et à l'anonymat. S'il était retenu dans le projet de loi en préparation, le concept d'information tel qu'il était défini dans le Livre blanc et qui englobait l'information sur support papier et électronique mais aussi celle sous toute autre forme, y compris verbale, allait bouleverser les méthodes de travail consacrées par l'usage.

Au sein du gouvernement, Jack Straw, ministre de l'Intérieur, avait pris la tête des réfractaires. Il s'opposait aux pouvoirs considérables accordés au commissaire à l'information et s'inquiétait des risques que la loi en gestation pourrait faire courir aux opérations menées par les services de police et de renseignements. D'autres responsables gouvernementaux, le secrétaire au Trésor en particulier, reprochait au projet son coût excessif, si bien que David Clark s'est vite retrouvé isolé malgré l'appui du Lord Chancellor, Lord Irvine. A la faveur du remaniement ministériel intervenu en juillet 1998, David Clark s'est trouvé évincé du Cabinet tandis que le projet de loi relatif au droit à l'information était transféré au ministère de l'Intérieur. 
39 Il a fallu attendre mai 1999 pour connaître la teneur du projet réécrit par Jack Straw. Si le Livre blanc avait suscité l'espoir, la nouvelle mouture rendue publique après un long temps de gestation souleva un véritable tollé. Un éditorialiste du Financial Times, d'ordinaire mesuré, pouvait ainsi écrire : «On attendait de M. Straw une charte du citoyen; il vient en fait d'exécuter un salut au secret ${ }^{20}$. Pour sa part, Maurice Frankel, directeur de la Campaign for Freedom of Information, ne cachait pas sa déception : «Plus on lit le projet de loi et plus on trouve de mérites au code [d'accès à l'information] des conservateurs» jugé sur certains points plus libéral que le nouveau texte travailliste ${ }^{21}$.

40 Parmi les dispositions les plus contestées du projet de loi figurait la modification $\mathrm{du}$ critère d'exemption de l'application du principe de communication de l'information. Là où le Livre blanc proposait que le refus de divulgation soit justifié par la présomption d'atteinte (harm) ou d'atteinte grave (substantial harm), selon les cas, à l'intérêt public, le projet de loi s'en tenait à la notion de prejudice. Il suffisait que le détenteur de l'information requise ait quelque raison de penser que sa divulgation était de nature à compromettre (prejudice) telle catégorie d'intérêts nationaux ou telle fonctionnalité de l'autorité publique sollicitée pour que la rétention d'information soit protégée par la loi. Se trouvaient ainsi soustraites du champ d'application du projet de loi les informations susceptibles de compromettre les intérêts économiques du Royaume-Uni ou de l'une de ses composantes ${ }^{22}$. Si les services de police n'échappaient pas au cadre de la loi, les actions relevant de la conduite d'enquêtes n'étaient pas susceptibles de faire l'objet de communications autres que celles décidées par les responsables. La même exemption protégeait les informations relatives aux enquêtes concernant les accidents - on pense ici aux nombreuses catastrophes ferroviaires - et les carences dans l'accomplissement de sa tâche par un membre d'une autorité publique ; comment ne pas évoquer à cette occasion la gestion des crises sanitaires?

41 C'est au total vingt-et-une catégories d'informations qui se trouvaient, de droit, exemptées contre sept prévues par le Livre blanc. Parmi celles-ci, les informations ayant pour origine les services de renseignement, les forces spéciales, les tribunaux d'exception les concernant - toutes institutions exclues du champ d'application du projet de loi étaient interdites de communication dès lors qu'un ministre de la Couronne pouvait en attester la provenance au moyen d'un certificat.

42 Plus restrictif que le Livre blanc, le projet de loi rendu public en mai 1999 réduisait aussi sensiblement les pouvoirs du commissaire à l'information dont les fonctions allaient être fusionnées avec celles du commissaire à la protection des données personnelles. Responsable du bon fonctionnement de la loi et de l'application scrupuleuse du code de bonne pratique promis par le gouvernement, il ne serait plus habilité à imposer à une autorité publique la divulgation d'une information au nom de l'intérêt public. C'est à l'organisme lui-même, éventuellement après intervention du commissaire, qu'il appartiendrait en dernier ressort d'apprécier l'opportunité de rendre publique ou non telle information sollicitée par un demandeur. La fonction d'arbitrage serait exercée par un tribunal spécifique, le projet de loi prévoyant toutefois une possibilité d'appel devant les juridictions traditionnelles.

43 Patrick Birkenshaw résume d'une phrase la réaction générale après la publication du texte du projet: "On a eu le sentiment que le projet de loi était le produit d'un pacte entre les forces des ténèbres au gouvernement et un rédacteur extrêmement astucieux, pourtant pas très au fait du contexte en matière de transparence. $»^{23}$ 
44 Jack Straw pouvait toutefois faire une lecture plus flatteuse de son texte dont les aspects positifs étaient souvent laissés dans l'ombre par les déçus du Livre blanc. Le champ d'application du projet de loi était plus large que celui du Livre blanc et incluait les activités commerciales et contractuelles qui n'étaient pas couvertes par le code de 1994. L'action des services de police, du moins sa partie administrative, était réintégrée parmi les fonctions et services auxquels s'appliquait la loi. Le gouvernement s'engageait par ce texte à abroger les nombreuses clauses de non communication d'informations figurant dans les lois, décrets et règlements antérieurs à l'exclusion, naturellement, de ceux relatifs à la protection de certaines informations et données (Official Secrets Act, Data Protection Act). Le projet de loi avait également pour mérite d'harmoniser la réglementation régissant l'accès à l'information et celle relative à la protection des données personnelles ${ }^{24}$. En confiant au responsable de la protection des données personnelles les missions assignées au commissaire à l'information, les concepteurs du projet combinaient adroitement des domaines d'action complémentaires. Enfin, les coûts d'accès à l'information supportés par le demandeur restaient raisonnables : $10 \%$ du coût réel de mise à disposition assortis d'un plafond de cinquante livres.

\section{Les concessions}

45 Le ministre de l'Intérieur savait pertinemment que l'examen du projet de loi par les commissions parlementaires ne serait pas une simple formalité. Les associations militantes - celle des consommateurs, Charter 88, ou Campaign for Freedom of Information, notamment - faisaient pression sur les représentants au Parlement pour obtenir une révision du texte. Très en pointe sur ces questions, le Guardian lançait dès juin 1999 une campagne de sensibilisation intitulée Open Up qui contribuait à entretenir la mobilisation.

46 Il n'est pas douteux que les objections formulées lors des séances de la commission de l'administration publique (Public Administration Committee) aient amené Jack Straw à opérer une retraite stratégique sur certains points, sans pour autant céder sur l'essentiel. Il a ainsi accepté l'ouverture des dossiers relatifs aux accidents, aux questions sanitaires et à la sécurité sur les lieux de travail sauf dans les cas où une action judiciaire serait engagée. Fut également supprimé du texte l'article dit «mosaïque » qui autorisait la rétention d'une information dès lors que celle-ci, corrélée avec une autre légalement accessible, risquait de porter atteinte à un type d'information protégée. Le raccourcissement du délai de communication de quarante à vingt jours ouvrables allait également dans le sens de l'apaisement.

47 Jack Straw a cependant tenu bon sur deux points fondamentaux. S'il a concédé une légère extension des prérogatives du commissaire désormais habilité à recommander la divulgation d'informations jugées non communicables par une autorité publique en vertu de la capacité d'interprétation qui lui est reconnue, il n'aura pas le pouvoir de faire prévaloir son avis ainsi que le prévoyait le Livre blanc qui avait toutefois subordonné ce pouvoir discrétionnaire à l'approbation du tribunal compétent dans ce domaine. Le commissaire peut néanmoins enjoindre (give notice) à une autorité publique de se conformer à l'article 17 de la loi qui autorise l'autorité concernée à refuser de faire savoir si elle dispose ou non d'une information requise au motif que ladite information est exemptée. L'autorité est, dans ce cas, tenue de préciser au requérant la nature de l'exemption invoquée. Le tribunal de l'accès à l'information (Information Tribunal) pourra 
éventuellement trancher le litige opposant le requérant à l'autorité ou l'autorité au commissaire.

48 Le ministre de l'Intérieur n'a rien lâché non plus sur la question de la protection de l'information touchant l'élaboration des politiques gouvernementales, la communication entre les différents rouages de l'appareil gouvernemental et les relations entre ministres et hauts fonctionnaires (article 35). «Toute organisation a besoin d'un minimum de discrétion pour pouvoir débattre des problèmes en toute franchise » explique Jack Straw ${ }^{25}$. Tel n'est pas l'avis de Patrick Birkenshaw, professeur de droit, qui estime que ce type d'exemption " affaiblit le droit à la liberté d'expression. [...] Si nous ne disposons pas des instruments nécessaires pour critiquer les orientations politiques, nos critiques ne peuvent avoir qu'une portée réduite. $»^{26}$ Ce n'est pas la faculté laissée par la loi aux dirigeants politiques et administratifs de rendre publiques après coup les données factuelles et statistiques ayant servi à l'élaboration des politiques qui suffira à renforcer chez le citoyen le sentiment de participation aux affaires du pays.

\section{Conclusion}

Devant la Société des directeurs de rédaction (Society of Editors), Chris Smith, alors ministre en charge de la Culture, des Médias et des Sports, déclarait que la loi sur la liberté d'information, finalement adoptée en avril 2000, constituait «un modeste pas en avant $~^{27}$, tandis que le chef de file de l'opposition, William Hague, promettait une révision radicale du texte dans le sens d'une plus grande transparence.

Certes, la loi n'a pas tenu toutes les promesses du Livre blanc. Elle n'en constitue pas moins une avancée considérable par rapport au code de 1994 qu'elle est appelée à remplacer. Par l'ampleur de son champ d'application d'abord qui englobe plus de 5000 autorités et organisations publiques et parapubliques en Angleterre, au pays de Galles et en Irlande $\mathrm{du} \mathrm{Nord}^{28}$. On ne peut ensuite tenir pour négligeable le fait que le texte constitutionnalise en quelque sorte le droit à l'information que les tribunaux sont, en dernier recours, appelés à faire respecter. Il reste à voir comment fonctionnera dans la réalité quotidienne cette immense ambition dont les contraintes, enfin mesurées, ont amené le gouvernement à retarder la traduction concrète. Si le calendrier échelonné de mise volontaire dans le domaine public des informations de base détenues par les différentes catégories d'autorités publiques sera bien respecté - l'opération s'étagera de novembre 2002 à juin 2004 - le dispositif central de la loi, à savoir l'accès à l'information par simple requête, n'entrera pas en vigueur avant janvier 2005. Ainsi en a décidé le gouvernement sous la pression de l'administration centrale qui mesure chaque jour davantage l'énormité de la tâche à laquelle elle va être confrontée. Certains voient dans ce report un signe supplémentaire de la tiédeur du premier ministre à l'égard d'une réforme qu'il présentait pourtant, naguère, comme annonciatrice d'une nouvelle relation entre le gouvernement et le peuple enfin associé à la gestion des affaires du pays. 


\section{NOTES}

1. British Public Opinion, Vol. XXIII, no 3, avril 2000.

2. Trevor BARNES, Open Up !, Fabian Tract 467, mars 1980, p. 10.

3. Rapporté par Trevor Barnes, ibid., p. 9.

4. Report of the Fulton Committee on the Civil Service, Cmnd. 3698, Londres: HMSO, 1968.

5. Departmental Committee on Section 2 of the Official Secrets Act, Cmnd. 5104, Londres : HMSO, 1972.

6. Données citées par Trevor BARNES, op.cit., p. 3.

7. Sa circulaire de 1977 est passée à la postérité sous l'appellation de « circulaire Croham».

8. Freedom of Information, Justice, 1978.

9. Official Information Bill, 1978.

10. K. G. ROBERTSON, Public Secrets. A Study in the Development of Government Secrecy, Londres : Macmillan, 1982, p. 89.

11. Green Paper on Open Government, Cmnd. 7520, Londres : HMSO, 1979.

12. Ibid., p. 3.

13. Rapporté par Patrick BIRKENSHAW, Reforming the Secret State, Milton Keynes : Open University Press, 1990, p. 9.

14. Patrick BIRKENSHAW, op.cit., p. 46.

15. Chiffres cités par Patrick BIRKENSHAW, Freedom of Information, Londres : Butterworths, 2001, p. 247.

16. Your Right to Know: Freedom of Information, Cmnd. 3818, Londres : The Stationary Office, 1997.

17. Exception faite de l'action de la police.

18. Il peut s'agir d'un particulier mais aussi d'une entreprise ou d'une association.

19. Conference on the Freedom of Information White Paper, organisée par la Campaign for Freedom of Information, 2 février 1998.

20. Philip STEPHENS in The Financial Times, 28 mai 1999.

21. Maurice FRANKEL, “Abysmal Handiwork”, The Guardian, 25 mai 1999.

22. Article 24 de l'avant-projet de loi Draft Freedom of Information Bill, Cmnd. 4355, Londres : The Stationary Office, mai 1999, qui deviendra l'article 27 dans la rédaction du projet présenté au Parlement en novembre 1999.

23. Patrick BIRKENSHAW, Freedom of Information, op.cit., p. 304.

24. Le gouvernement a été amené à réviser en 1998 le Data Protection Act adopté en 1984 pour le mettre en conformité avec une directive européenne plus contraignante promulguée en 1995.

25. Jack STRAW, “A Balance of Rights”, Press Gazette, 24 novembre 2000.

26. Patrick BIRKENSHAW, Freedom of Information, op.cit., p. 470.

27. Press Gazette, 20 octobre 2000.

28. L'Écosse est en passe de se doter d'une législation spécifique dans ce domaine. 


\section{RÉSUMÉS}

Menée en parallèle au combat visant la réforme de la loi sur les secrets officiels, l'action engagée depuis les années soixante-dix pour faire reconnaître par le Parlement le droit à l'information s'est heurtée à l'inertie naturelle des gouvernements qui ne manquaient pourtant pas, avant chaque élection, d'affirmer la reconnaissance de ce droit comme une priorité démocratique. La déception qui a accueilli le projet de loi présenté en 1999 par le gouvernement Blair était à la mesure des espoirs suscités par le Livre blanc publié deux ans auparavant. Les concessions accordées par le Cabinet ont permis l'adoption d'un texte qui constitue, au total, une avancée significative en matière d'accès à l'information. Encore faudrait-il que la loi entre, enfin, en vigueur.

Reforming the Official Secrets Act was also on the agenda of those who had been campaigning for Parliament to recognize the right to access official information. Their efforts had been thwarted by the natural inertia of successive governments who, however, invariably declared during each general election campaign that Freedom of Information was the cornerstone of democracy. The disappointment which met the FOI Bill presented by the Blair Government in 1999 reflected the frustration of those who had pinned their hopes on the White Paper published two years earlier. In its amended version, following concessions by the Cabinet, the new FOI Act marks, on the whole, a significant improvement on the previous situation. However, its delayed enforcement is a major cause for concern.

\section{AUTEUR}

\section{JEAN-CLAUDE SERGEANT}

Université Paris III / Maison française d'Oxford 